\title{
Linfedema de extremidades inferiores secundario al tratamiento oncológico: actualización en el diagnóstico y tratamiento quirúrgico
}

\author{
Nicolás Pereira C. ${ }^{1,2}$ y Takumi Yamamoto ${ }^{3}$
}

'Departamento de Cirugía Plástica, Clínica Las Condes. Santiago, Chile.

2Departamento de Cirugía Plástica y Quemados, Hospital

del Trabajador. Santiago

Plastic and Reconstructive

Surgery Department, Center

Hospital of the National

Center for Global Health and

Medicine. Tokyo, Japan.

Recibido el 24 de enero

de 2018 y aceptado para publicación el 3 de mayo de

Correspondencia a: Dr. Nicolás Pereira C npereira@clc.c

\section{Lower extremity lymphedema secondary to oncological treatment: update on diagnosis and surgical treatment}

Lower extremities secondary lymphedema is the accumulation of protein-rich fluid in the interstitium as a consequence of the damage of lymphatic vessels. In our country, it is more frequently related to the surgical treatment of cancer. The incidence varies depending on the type of cancer and the treatment received, with an increased risk in cases in which lymphadenectomy and radiotherapy are performed. Clinical diagnosis and imaging techniques are essential to asses the functional status of the lymphatic system. The main objectives in the management of lymphedema are to limit patient morbidity, improve functionality and quality of life. There are procedures that seek to prevent the development of lower extremity lymphedema. Once established, the treatment can be conservative and surgical. Surgical treatment includes physiological (reconstructive) and excisional procedures. Success depends on a good selection of patients and the performance of an individualized treatment. The following is a review regarding the incidence, risk factors, diagnostic strategies and surgical techniques with emphasis on microsurgical treatment.

Key words: lymphedema; lymphadenectomy; indocyanine green lymphography; supermicrosurgery; lymphovenous anastomosis; vascularized lymph-node transfer.

\section{Resumen}

El linfedema secundario de extremidades inferiores es la acumulación de fluido rico en proteínas en el intersticio como consecuencia al daño de vasos linfáticos. En nuestro medio, se relaciona más frecuentemente al tratamiento quirúrgico del cáncer. La incidencia varía dependiendo del tipo de cáncer y el tratamiento recibido, existiendo mayor riesgo en los casos en los que se realiza linfadenectomía y radioterapia. El diagnóstico clínico y a través de técnicas de imágenes es fundamental para evaluar el estado funcional del sistema linfático. Los objetivos principales en el manejo del linfedema son limitar la morbilidad del paciente, mejorar la funcionalidad y la calidad de vida. Existen procedimientos que buscan prevenir el desarrollo del linfedema de extremidades inferiores. Una vez establecido, el tratamiento puede ser conservador y quirúrgico. El tratamiento quirúrgico incluye procedimientos fisiológicos (reconstructivos) y resectivos. El éxito depende de una buena selección de los pacientes y la realización de un tratamiento individualizado. A continuación se presenta una revisión en cuanto a la incidencia, factores de riesgo, estrategias diagnósticas y técnicas quirúrgicas con énfasis en el tratamiento microquirúrgico.

Palabras clave: linfedema; linfadenectomía; linfografía con verde indocianina; supermicrocirugía; anastomosis linfático-venosa; transferencia de linfonodos vascularizados. 


\section{Introducción}

El linfedema es la acumulación de fluido rico en proteínas en el intersticio, secundario a anomalías en el transporte linfático. Puede manifestarse como edema blando con fóvea que puede progresar a un edema sin fóvea y crecimiento irreversible de la extremidad, llevando a una fibrosis progresiva, obstrucción de los vasos linfáticos y celulitis a repetición ${ }^{1}$.

El linfedema secundario es consecuencia del daño de vasos linfáticos debido al trauma, cáncer, cirugía o infección. Las zonas más comprometidas son la extremidad inferior $(90 \%)$, extremidad superior $(10 \%)$ y genitales $(<1 \%)^{2}$. En nuestro medio se relaciona más frecuentemente al tratamiento quirúrgico del cáncer. En extremidades inferiores puede ocurrir secundario al tratamiento de varios tipos de cáncer como melanoma $16 \%$, ginecológico $20 \%$, genitourinario $10 \%$, cabeza y cuello $4 \%$ y sarcomas $30 \%{ }^{3}$.

Una característica común de los tumores sólidos es su patrón de diseminación a linfonodos regionales previo a las metástasis a distancia. Ante la ausencia de tratamientos sistémicos efectivos, los principios oncológicos para el tratamiento de metástasis regionales linfáticas incluye la disección del territorio de linfonodos involucrados, ya sea inguinofemorales o pélvicos (iliacos/obturadores). Si bien los tumores de partes blandas y osteosarcomas rara vez dan metástasis a los linfonodos regionales, producen obstrucción primaria o disrupción del sistema linfático (luego del tratamiento), resultando en linfedema secundario.

El linfedema de extremidades inferiores secundario al tratamiento oncológico, generalmente, se manifiesta dentro del primer año luego del tratamiento en el $80 \%$ de los casos ${ }^{4}$. A continuación se presenta una actualización en el diagnóstico y tratamiento quirúrgico del linfedema de extremidades inferiores secundario al manejo oncológico de diversos tipos de cáncer.

\section{Incidencia y factores de riesgo}

La incidencia de linfedema secundario de extremidades inferiores varía dependiendo del tipo de cáncer y el tratamiento recibido. Existen factores de riesgo asociados al desarrollo de linfedema de extremidades inferiores secundario al tratamiento oncológico, siendo la linfadenectomía y la radioterapia posoperatoria los más importantes para su aparición ${ }^{5}$. En el caso del melanoma, la incidencia de linfedema luego de la disección de linfonodos inguinofemorales es $18 \% \%^{3}$.

Con respecto al linfedema secundario al cáncer genitourinario, la incidencia de linfedema es $11 \%$. En el caso específico del cáncer de pene, próstata y cáncer de vejiga, la incidencia es $21 \%, 4 \%$ y $16 \%$ respectivamente ${ }^{3}$. El linfedema en el cáncer de próstata varía dependiendo del tipo de linfadenectomía (LND: lymph node dissection), siendo 0,6\%-1,4\% de los casos con LND limitada y en el 8,8\%-15,7\% de la extendida ${ }^{6}$.

La incidencia del linfedema asociado a cáncer ginecológico es aproximadamente $25 \%$, presentándose en el $27 \%$ de los casos de cáncer cérvico-uterino, $30 \%$ del cáncer vulvar y $4,7 \%$ del cáncer de ovarios $^{3}$. En un estudio retrospectivo, Hareyama et al. ${ }^{7}$ reportaron una incidencia de $21,8 \%$ de linfedema secundario a cáncer ginecológico, el cual aumenta con el tiempo: $12,9 \%$ al año; $20,3 \%$ a los 5 años y $25,4 \%$ a los 10 años de seguimiento.

Los pacientes con cáncer que requieren linfadenectomía para su tratamiento presentan mayor riesgo de desarrollar linfedema. En general, el 22\% de los pacientes con linfadenectomía pélvica presentan linfedema; melanoma con LND ilíaco/obturador en el $42 \%$; cáncer genitourinario en el $8 \%$ y cáncer ginecológico en el $27 \%{ }^{3}$. En el cáncer cérvico-uterino, la resección de los linfonodos circunflejos ilíacos se ha asociado a un mayor riesgo ${ }^{8}$.

El número de linfonodos resecados en el tratamiento del cáncer ginecológico como factor de riesgo es controversial. En el cáncer cérvico-uterino, Tanaka et al. ${ }^{9}$, demostraron que la incidencia de linfedema era $39,1 \%$ cuando el número de linfonodos resecados era mayor a 24 . Füller et al.$^{10}$, reportaron que cuando los linfonodos resecados eran mayor a 25 , la incidencia de linfedema fue $32,5 \%$. Por otro lado, Hareyama et al. $^{7}$, demuestran una relación inversa entre la cantidad de linfonodos resecados y linfedema, siendo mayor cuando el número era inferior a 70 . Kim et al. ${ }^{11}$, por su parte, establecen que no existe correlación entre el número de linfonodos resecados y el desarrollo de linfedema secundario a la LND en pacientes con cáncer cérvico-uterino.

La radioterapia adyuvante se considera un factor de riesgo para el desarrollo de linfedema de extremidades inferiores ${ }^{8,11}$. El 31\% de los pacientes que reciben radioterapia presentan linfedema; $50 \%$ de los pacientes con melanoma, $16 \%$ de los con cáncer genitourinario y $34 \%$ de los con cáncer ginecológico $^{3}$.

La obesidad juega un rol importante en el desarrollo de linfedema luego del cáncer de endometrio. Mendivil et al. ${ }^{12}$, sostienen que un índice de masa 
corporal $(\mathrm{IMC}) \geq 35 \mathrm{~kg} / \mathrm{m}^{2}$ influye significativamente para la manifestación de esta patología.

Diversos estudios han demostrado que los pacientes que desarrollan celulitis, presentan episodios recurrentes durante el primer año posterior a la cirugía. El deterioro del drenaje linfático causado por la celulitis a repetición produce una predisposición al desarrollo de linfedema.

\section{Diagnóstico}

La historia clínica es muy importante para iniciar el proceso diagnóstico. Se debe interrogar sobre antecedentes familiares, cirugía reciente, infecciones, exposición en viajes y episodios previos de síntomas. Específicamente en linfedema de extremidades inferiores secundario al tratamiento oncológico, se debe investigar el tipo de cáncer, cirugía practicada, realización y tipo de linfadenectomía, compromiso ganglionar, tratamientos adyuvantes (radio y/o quimioterapia), complicaciones, etc. Los síntomas comunes incluyen edema, pesadez, engrosamiento o firmeza de los tejidos, parestesias, infecciones recurrentes y ocasionalmente dolor.

En el examen físico, la medición volumétrica de la extremidad es lo principal para diagnóstico y seguimiento de la progresión. Si bien, existen numerosas formas disponibles para evaluar el linfedema (desplazamiento de agua, mediciones circunferenciales, perímetro y espectrometría de bioimpedancia), no hay un método universalmente aceptado. Una diferencia de circunferencia mayor a $2 \mathrm{~cm}$ o de volumen mayor a $200 \mathrm{cc}$ de la extremidad afectada con respecto a la extremidad sana, se considera significativa. Cuando se obtienen múltiples medidas de circunferencia, se puede calcular el volumen utilizando la fórmula del cono trunco (frustrum) ${ }^{13}$. El índice de linfedema de extremidad inferior ( $L E L$ index) es un método simple para determinar la severidad, el cual se correlaciona con la etapificación. Es muy útil para evaluar la progresión en el tiempo, la efectividad del tratamiento y para ser utilizado en casos bilaterales. Se obtiene luego de la sumatoria del cuadrado de las circunferencias en 5 áreas de la extremidad inferior, dividido por el $\mathrm{IMC}^{14}$.

Existe un creciente interés en detectar el linfedema en etapas subclínicas o iniciales (cambios de volumen relativo de 5\%-10\%), debido a que un diagnóstico temprano permite una intervención y tratamiento precoz obteniendo mejores resultados ${ }^{15}$. Una vez hecho el diagnóstico clínico y la etapificación según la Sociedad Internacional de Linfología (0-IV), se deben realizar estudios complementarios para determinar la extensión de la enfermedad y la funcionalidad del sistema linfático ${ }^{16}$.

La linfocintigrafía isotópica es el estudio más común y actualmente considerado gold standard para el diagnóstico de linfedema. Se realiza una inyección subdérmica de contraste no ionizante (Tc 99m, Tc 99m-HAS, o Tc 99m dextrán) en los espacios interdigitales del pie. La macromolécula que se sigue con gamma cámara, entrega una evaluación estática y dinámica del flujo linfático, evidenciando zonas de bloqueo y/o flujo reverso, además, de captación a nivel inguinal. El hallazgo linfocintigráfico de vasos linfáticos principales, sin colaterales, permite predecir una buena respuesta al tratamiento conservador con terapia descongestiva compleja en etapas iniciales.

La linforresonancia magnética (MRL) es capaz de mostrar cambios anatómicos detallados en los vasos linfáticos y linfonodos. Permite visualizar claramente vasos linfáticos colectores tortuoso y dilatado en extremidades linfoedematosas. Proporciona información sobre el status funcional del transporte de linfa en los vasos linfáticos y en linfonodos mediante la visualización del flujo linfático contrastado en los canales linfáticos y dentro de los linfonodos.

En la linfografía con verde indocianina (ICG) se realiza una inyección subdérmica de ICG en los espacios interdigitales del pie y se observa mediante una cámara de fluorescencia cercana al infrarrojo (ej. SPY ${ }^{\circledR}$, PDE-neo $\left.{ }^{\circledR}\right)$. Permite evaluar el sistema linfático en tiempo real, identificando la presencia y ubicación de vasos linfáticos permeables. Es preciso para estadificar el linfedema según su funcionalidad en patrón lineal (normal) y patrón de dermal backflow (anormal: splash, stardust, difuso) (Figura 1). Este último, se divide a su vez, según la zona anatómica comprometida (Leg dermal backflow (LDB): I inguinal, II muslo, III pierna y IV tobillo/ pie $)^{17}$. La presencia de patrón splash es un hallazgo temprano de disfunción linfática en la extremidad inferior asintomática del paciente con linfedema secundario ${ }^{18}$. Permite orientar en la indicación del manejo quirúrgico ${ }^{19}$.

\section{Tratamiento}

El tratamiento del linfedema de extremidades inferiores secundario al tratamiento oncológico debe estar a cargo de un equipo multidisciplinario. Es particularmente complejo debido a la posición dependiente de la gravedad de las piernas, el mayor volumen y el efecto de la deambulación. Los objetivos principales del manejo son limitar la morbilidad del paciente, mejorar la funcionalidad y la calidad 
de vida. Con el fin de lograr esto, se debe disminuir el edema, mejorar la higiene, reducir las tasas de infección, mejorar la movilidad y disminuir la carga de las terapias diarias. Por lo tanto, estos factores se deben tener en cuenta al momento de evaluar los resultados del tratamiento.

Existen procedimientos que buscan prevenir el desarrollo del linfedema de extremidades inferiores. Una vez establecido, el tratamiento puede ser conservador y quirúrgico. El tratamiento quirúrgico, a su vez, incluye procedimientos fisiológicos (reconstructivos) y resectivos.

\section{Tratamiento preventivo}

La prevención del linfedema de extremidades inferiores secundario al tratamiento oncológico ha sido un tema de interés en los últimos años. Benoit et al. ${ }^{20}$, reportan el uso de un colgajo de omento pediculado en la región inguinal luego de la LND ilioinguinal para la prevención del linfedema. Sostienen que el colgajo facilitaría la absorción o transporte de linfa disminuyendo la incidencia de linfedema de extremidades inferiores, incluso, posterior a radioterapia, presentando resultados promisorios.

El abordaje linfático microquirúrgico preventivo curativo (LYMPHA, por sus siglas en inglés-Lymphedema Microsurgical Preventive Healing Approach) es una técnica quirúrgica de prevención primaria de linfedema luego de la LND ilio-inguinal ${ }^{21,22}$. Consiste en la realización de anastomosis linfático-venosas múltiples (MLVA) al momento de la disección ilio-inguinal, entre los linfáticos seccionados que drenan la extremidad inferior y ramas colaterales de la vena safena. Está indicado en pacientes con cáncer de vulva, melanoma del tronco $u$ otro cáncer que requieren LND inguinofemoral, pero que no se encuentran en las extremidades, siendo esta última una contraindicación por razones oncológicas. En un seguimiento a 26 meses (6-36 meses) de 27 pacientes (11 cáncer de vulva y 16 melanoma), sólo 1 paciente presentó linfedema ${ }^{22}$.

Los pacientes que desarrollan linfedema en una extremidad secundario al tratamiento del cáncer ginecológico tienen mayor riesgo de desarrollar linfedema en la extremidad contralateral ${ }^{7}$. Además, se ha visto que la extremidad sana puede presentar alteración en la función linfática previo a la manifestación de sintomatología ${ }^{17}$. Teniendo esto en consideración, se ha propuesto la realización de múltiples anastomosis linfático-venosas (LVA) a nivel del tobillo como tratamiento preventivo de linfedema de la extremidad asintomática, en pacientes con linfedema secundario unilateral debido

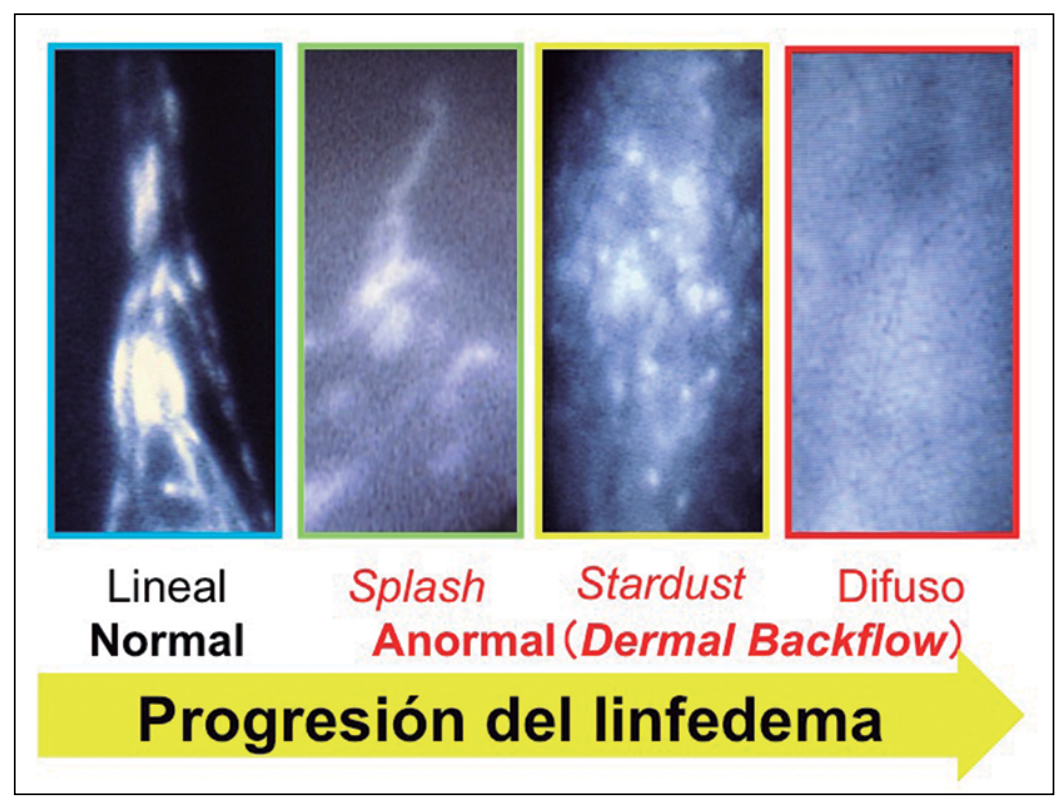

Figura 1. Tipos de patrones en linfografía con ICG que indican el grado de disfunción linfática.

a la posibilidad de desarrollar esta enfermedad en el futuro ${ }^{23}$. Aún se necesitan estudios a largo plazo para evaluar su real utilidad.

La LVA de los vasos linfáticos eferentes de los linfonodos inguinales es un tratamiento preventivo en pacientes con linfedema subclínico de extremidades inferiores secundario a la linfadenectomía pelviana por tratamiento oncológico. El diagnóstico del status subclínico ( $L D B I)$ se realiza mediante linfografía con ICG, en pacientes con el antecedente quirúrgico y sin síntomas de linfedema. Consiste en la exploración de linfonodos de la región inguinal, identificación de vasos eferentes y realización de $\mathrm{LVA}^{24}$.

\section{Tratamiento conservador}

Tradicionalmente, el tratamiento conservador es el pilar fundamental y la forma inicial de tratamiento del linfedema. La terapia descongestiva compleja (TDC) debe ser realizada por un profesional especializado en linfedema, siendo más efectiva en etapas tempranas ${ }^{25}$. Tiene una fase inicial reductiva (3-8 semanas) que consiste en el drenaje linfático manual, vendaje multicapa, ejercicios terapéuticos, cuidado de la piel, educación y autocuidado, compresión elástica; y una fase de mantenimiento que contempla el autodrenaje linfático permanente, ejercicios, cuidado de la piel y uso de prendas/vendaje de compresión ${ }^{25}$.

En estudios sobre linfedema secundario al trata- 
miento ginecológico, la TDC logra un 43,5\%-55,1\% de reducción del porcentaje de exceso de volumen y mejora significativamente la calidad de vida ${ }^{26}$. Si bien son efectivas, estas terapias tienen sus detractores. No solamente debido a que el tratamiento debe ser indefinido para mantener su eficacia, sino porque existe una amplia variabilidad en la calidad de los tratamientos y de las prendas compresivas.

\section{Tratamiento quirúrgico}

\section{Procedimientos fisiológicos o reconstructivos}

Actualmente, los procedimientos fisiológicos más comúnmente realizados son la anastomosis linfáticovenosas y la transferencia de linfonodos vascularizados. El injerto autólogo de vasos linfáticos no ha demostrado resultados reproducibles por lo que no se utiliza ampliamente.

\section{Anastomosis linfático-venosas (LVA)}

Esta técnica tiene como objetivo redirigir el exceso de fluido linfático a la circulación venosa y así superar zonas de obstrucción. La gradiente de presión linfático-venosa y la presencia de válvulas competentes en las venas permite el flujo linfático a través de la anastomosis.

No hay consenso respecto al momento de la cirugía, el número, localización y configuración de las LVA. Mihara et al. ${ }^{27}$, establecen que existe una relación directa entre el número de LVA y la reducción del volumen, mientras que Seki et al. ${ }^{28}$, sugieren que basta con una LVA funcionante para obtener resultados satisfactorios.

La técnica consiste en la inyección subdérmica

Figura 2. Anastomosis linfático-venosa para el tratamiento del linfedema de extremidades inferiores con técnica de supermicrocirugía (vasos de 0,5 mm). Asterisco, vena; flecha, vaso linfático. de ICG en los espacios interdigitales del pie y búsqueda mediante una cámara cercana al infrarrojo la presencia de vasos linfáticos funcionales, los cuales se marcan. Este mapeo se debe correlacionar con los hallazgos de la MRL, en caso de disponer de ella. Se realiza una incisión transversal para identificar y aislar un vaso linfático y su vénula adyacente. Se realiza un bypass (LVA) utilizando nylon 11-0 y 12-0 con aguja 50-65 um, ya sea término-terminal o latero-término (Figura 2). Una LVA es más simple y efectiva mientras más precoz se realice ${ }^{29}$.

Diversos estudios han demostrado la eficacia de la LVA en el tratamiento del linfedema. En pacientes con linfedema inicial, que presentan recientemente patrón stardust, la realización de LVA elimina la necesidad de tratamiento compresivo en un $44,8 \%$ de los casos. Koshima et al. ${ }^{30}$, compararon 12 pacientes con linfedema de extremidades inferiores que siguieron tratamiento conservador versus 13 pacientes en los cuales se realizó LVA más tratamiento conservador. En el grupo control, el seguimiento promedio fue de 1,5 años y la disminución de la circunferencia fue $0,6 \mathrm{~cm}$ ( $8 \%$ del exceso); mientras que en el segundo grupo el seguimiento fue de 3,3 años y la disminución de la circunferencia fue 4,7 cm (55,6\% del exceso).

Mihara et al. ${ }^{27}$, evaluaron el resultado de la LVA en 162 extremidades inferiores operadas. Con respecto a la disminución de la circunferencia, un $47,7 \%$ presentó mejoría, $27,3 \%$ se mantuvo estable y un $25 \%$ empeoró. Desde el punto de vista subjetivo, un $61,5 \%$ presentó mejoría en la sintomatología, $34,9 \%$ no tuvo cambios y un 3,7\% refirió haber empeorado. Por otro lado, los episodios de celulitis también disminuyeron desde 0,89 episodios por año en el preoperatorio a 0,13 . Hubo una asociación directa entre el número de LVA y la cantidad de la reducción de volumen.

Seki et al. ${ }^{28}$, mediante la técnica de LVA en la cara medial del muslo a nivel del borde superior de la rótula (SEKI-superior edge of the knee incision), logró mejores resultados en cuanto al índice de severidad del linfedema de extremidad inferior al compararlo con el grupo con múltiples abordajes no-SEKI.

Otros estudios han corroborado estos hallazgos, complementando a la disminución del volumen, una disminución de las tasas de infección y la suspensión de las prendas de compresión después de $\mathrm{LVA}^{31}$. Las complicaciones de la LVA son inusuales y mínima, incluyendo infección, fístula linfática y problemas de cicatrización de la herida. Los beneficios de la técnica son lo mínimamente invasivo, escaso dolor y posibilidad de alta a las 24-48 horas. 
Transferencia de linfonodos vascularizados (VLNT)

Descrito inicialmente por Becker et al. ${ }^{32}$, para el tratamiento del linfedema, es un procedimiento que consiste en la transferencia de un colgajo libre de linfonodos a la extremidad afectada mediante microcirugía (Figura 3).

Las teorías que explican el mecanismo de acción de VLNT son que los linfonodos trasferidos actuarían como una "esponja o bomba" que atrae linfa y pasa al sistema venoso mediante conexiones linfático-venosas naturales dentro del colgajo ${ }^{33} \mathrm{y} / \mathrm{o}$ a través de linfoangiogénesis en el lecho receptor mediada por factor de crecimiento vascular endotelial C (VEGF-C) que estimula el crecimiento e inosculación de vasos linfáticos a la red linfática de los VLNT ${ }^{34}$.

Para el tratamiento del linfedema de extremidades inferiores secundario al tratamiento oncológico, los linfonodos más utilizados son los supraclaviculares, submentales, torácicos laterales y gastroepiploicos.

Cuando se utilizan los linfonodos torácicos laterales (axilares) existe un riesgo potencial de linfedema iatrogénico de la zona dadora. El "mapeo linfático reverso" permite diferenciar los linfonodos que drenan la extremidad superior (torácicos) con un radionucleótido marcado, de los linfonodos que drenan el tórax con $\mathrm{ICG}^{35}$. Aunque el riesgo de linfedema iatrogénico de la zona dadora luego de VLNT es bajo, es un riesgo real y debe ser discutido con el paciente.
La selección de la zona receptora se basa en la localización del linfedema, disponibilidad de vasos receptores, cicatrices previas, apariencia estética y la experiencia del cirujano. Las zonas receptoras descritas son la región inguinal, zona poplítea y tobillo. Cuando se propone la ingle luego de una cirugía previa en la zona, se debe remover todo el tejido cicatricial hasta plano vital y reemplazarlo por un colgajo bien vascularizado. Además, los colgajos en esta ubicación se pueden ocultar de mejor manera y otorgar un mejor resultado estético. Por el contrario, las zonas distales como receptora de VLNT son más fáciles de disecar y aportarían el efecto "esponja" como mecanismo de drenaje, por lo que en teoría comenzarían a funcionar inmediatamente.

Diversos estudios han reportado reducciones significativas en la circunferencia o el volumen entre un $35 \%$ a $67 \%$ luego de VLNT ${ }^{36,37}$. En la mayoría de los casos se trata de estudios retrospectivos, sin embargo, Patel et al. ${ }^{36}$, reportaron un $35,2 \%$ de reducción de la circunferencia de la extremidad inferior afectada con respecto a la sana, en un estudio prospectivo de 10 pacientes con seguimiento a 12 meses.

En un estudio retrospectivo, Batista et al. ${ }^{38}$, utilizando principalmente linfonodos torácicos laterales, muestra una reducción de un 46,3\% del exceso de volumen con mejores resultados en los casos que tenían menor exceso de volumen preoperatorio. Cheng et al. ${ }^{37}$, transfiriendo linfonodos submentales al nivel del tobillo, obtiene reducciones de la circunferencia en la extremidad afectada con respecto a la

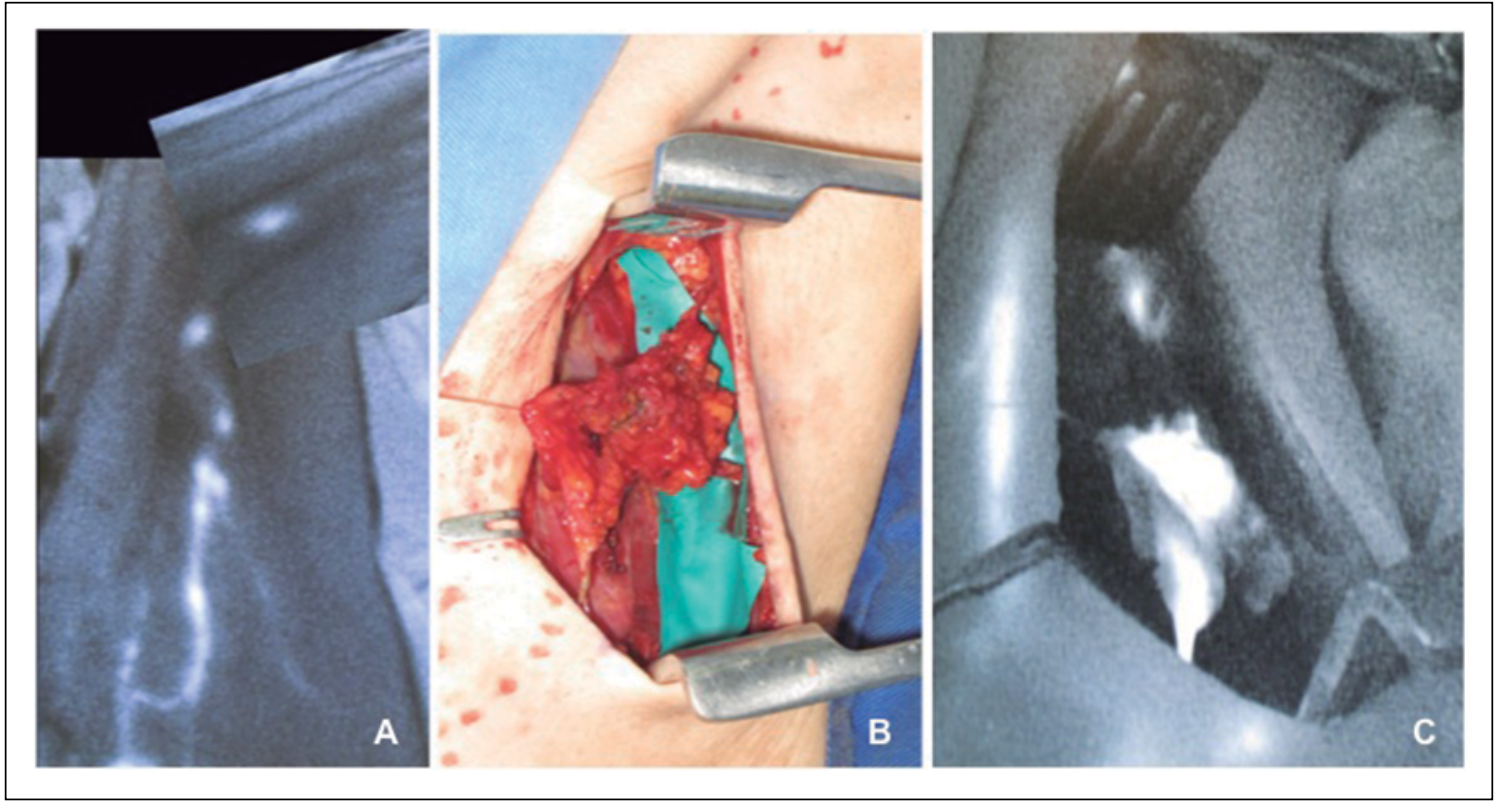

Figura 3. Transferencia de linfonodos vascularizados para el tratamiento del linfedema de extremidades inferiores. A) Linfografía con ICG en la pared torácica que muestra el drenaje a los linfonodos torácicos laterales; B) Linfonodos torácicos laterales in situ en la zona dadora; C) Linfografía con ICG que muestra fluorescencia en los linfonodos torácicos laterales previos a ser transferidos a la extremidad inferior. 
extremidad sana de $64 \%$ sobre la rodilla, $63,7 \%$ bajo la rodilla y $67,3 \%$ sobre el tobillo.

Las complicaciones de la zona receptora pueden ser la pérdida parcial/total del colgajo, pérdida parcial del injerto de piel, infecciones de la herida operatoria, retardo en la cicatrización y edema prolongado del colgajo. En la zona dadora, pueden ocurrir complicaciones de la herida, linforrea, linfocele y linfedema iatrogénico (teóricamente más frecuente en linfonodos torácicos laterales).

\section{Procedimientos resectivos}

El procedimiento de Charles consiste en escisión radical circunferencial del tejido linfoedematoso hasta la fascia profunda y cobertura inmediata con injerto dermoepidérmico de la piel resecada. Debido a lo desfigurante del procedimiento, está indicado solamente para los casos más severos. El procedimiento de Charles modificado utiliza terapia con presión negativa después de la resección inicial, realizando el injerto a los 5 a 7 días. Se ha propuesto la realización simultánea de un procedimiento resectivo tipo Charles, en que se preserva el sistema venoso superficial y se transfiere un colgajo de ganglios linfáticos al tobillo ${ }^{39}$.

La reducción radical del linfedema con preser- vación de perforantes (RRPP) se basa en un mejor conocimiento de la irrigación de la piel, permitiendo una resección extensa manteniendo la perfusión. Esta técnica ha mostrado resultados considerables, siendo particularmente útil en linfedema de extremidades inferiores.

La liposucción remueve el tejido graso infiltrado que no responde a TCD o a procedimientos fisiológicos, pero lamentablemente no elimina la necesidad de tratamiento compresivo continuo en el posoperatorio ${ }^{40}$. A pesar de estas deficiencias, la liposucción ha demostrado disminuir el edema, las tasas de infección en la extremidad linfoedematosa, por lo que continúa siendo una herramienta muy útil para el tratamiento del linfedema severo y como técnica complementaria.

\section{Enfrentamiento terapéutico del paciente con linfedema de extremidades inferiores secundario al tratamiento oncológico}

Se debe realizar una evaluación completa de manera de poder ofrecer un tratamiento individualizado (Figura 4). El tratamiento se basa en la etapa clínica, consideraciones anatómicas/funcionales y el deseo
Figura 4. Algoritmo general para el tratamiento de la paciente con linfedema de extremidades inferiores secundario al tratamiento oncológico. EEIl, extremidades inferiores; ICG, verde indocianina; MRL, linforresonancia magnética; LVA, anastomosis linfático-venosas; LN-VA, anastomosis linfonodo venosa; VLNT, transferencia de linfonodos vascularizados.

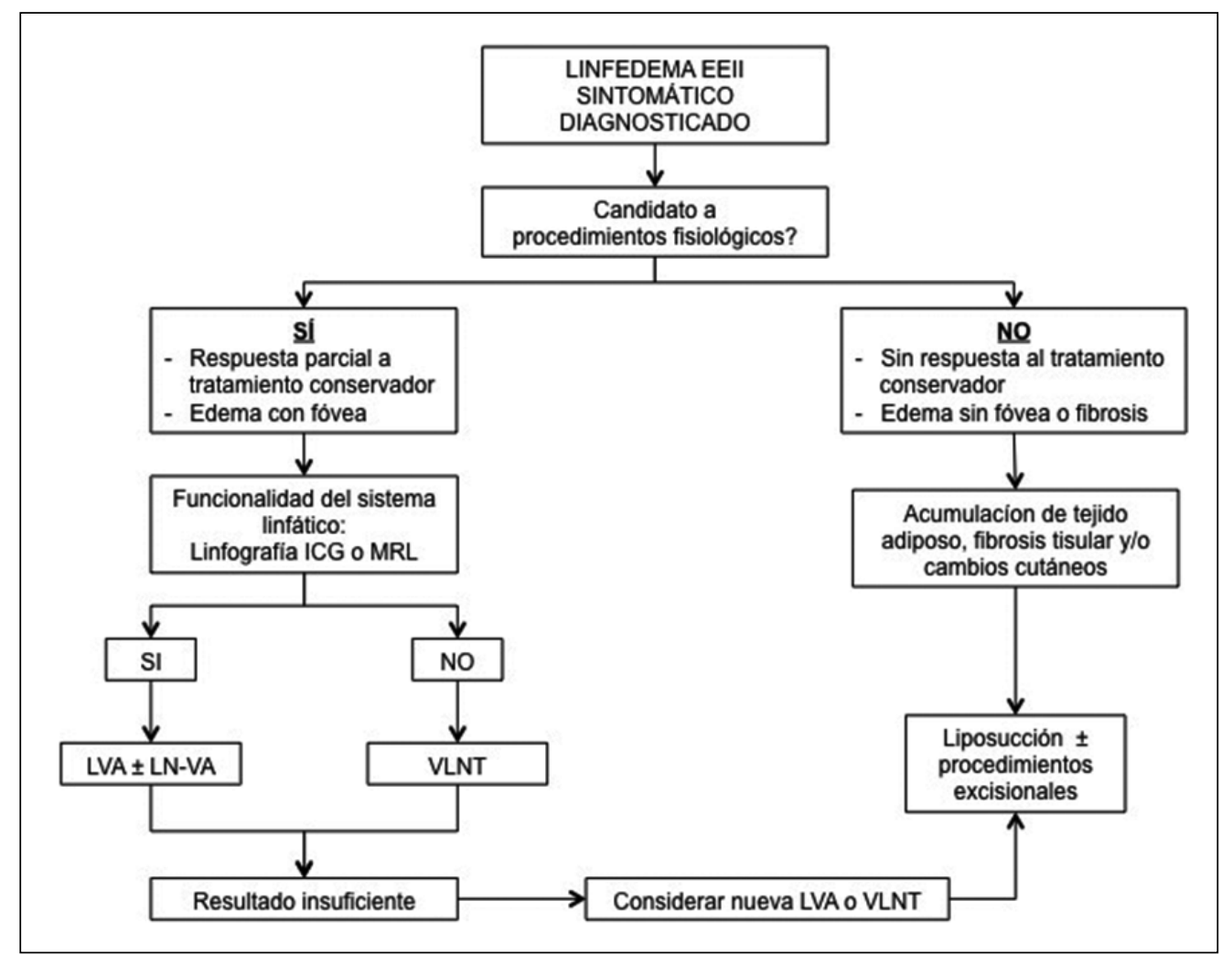


del paciente. Los casos que presentan edema con fóvea y respuesta parcial al tratamiento conservador, son candidatos para procedimientos fisiológicos ya que el edema se debe principalmente a la acumulación de fluido linfático que puede ser resuelto mediante LVA o VLNT. En casos más avanzados, el aumento de volumen se debe a la acumulación de tejido adiposo, por lo que son candidatos a procedimientos resectivos (liposucción, RRPP o Charles modificado).

Para decidir qué procedimiento fisiológico realizar, se debe hacer el diagnóstico con linfocintigrafía y evaluar la funcionalidad del sistema linfático mediante MRL y/o linfografía con ICG. Si el sistema linfático aún es funcional, el paciente es candidato para LVA con/sin LN-VA dependiendo si existe captación de los linfonodos inguinales en la linfocintigrafía. Si el sistema linfático es disfuncionante, se propone la realización de VLNT.

Cuando se realiza una VLNT, el lecho receptor se debe preparar resecando todo el tejido cicatricial, liberar la compresión venosa en caso que la zona receptora sea el sitio de la cirugía previa y/o radioterapia, y así permitir la linfangiogénesis entre el colgajo y el lecho receptor. En los casos con exceso de adiposidad subcutánea residual, se pueden realizar procedimientos resectivos 6-12 meses después de la primera cirugía.

Durante el período posoperatorio, los pacientes deben discontinuar la terapia física y reasumir progresivamente sus actividades normales según tolerancia. El uso de prendas elásticas en el período posoperatorio es controversial, pero en general se debe reasumir inmediatamente después de LVA y suspender por 2-4 semanas en el caso de VLNT. Luego del período posoperatorio inicial, los pacientes deben controlarse periódicamente. Los resultados se evidencian desde los 3 meses y se estabilizan al año posoperatorio. Los resultados se evalúan de acuerdo a la mejoría subjetiva del paciente, medidas perimetrales y volumétricas, frecuencia de episodios de celulitis y cuestionarios de calidad de vida (Figura 5).

\section{Conclusiones}

El linfedema de extremidades inferiores secundario al tratamiento oncológico es muy frecuente

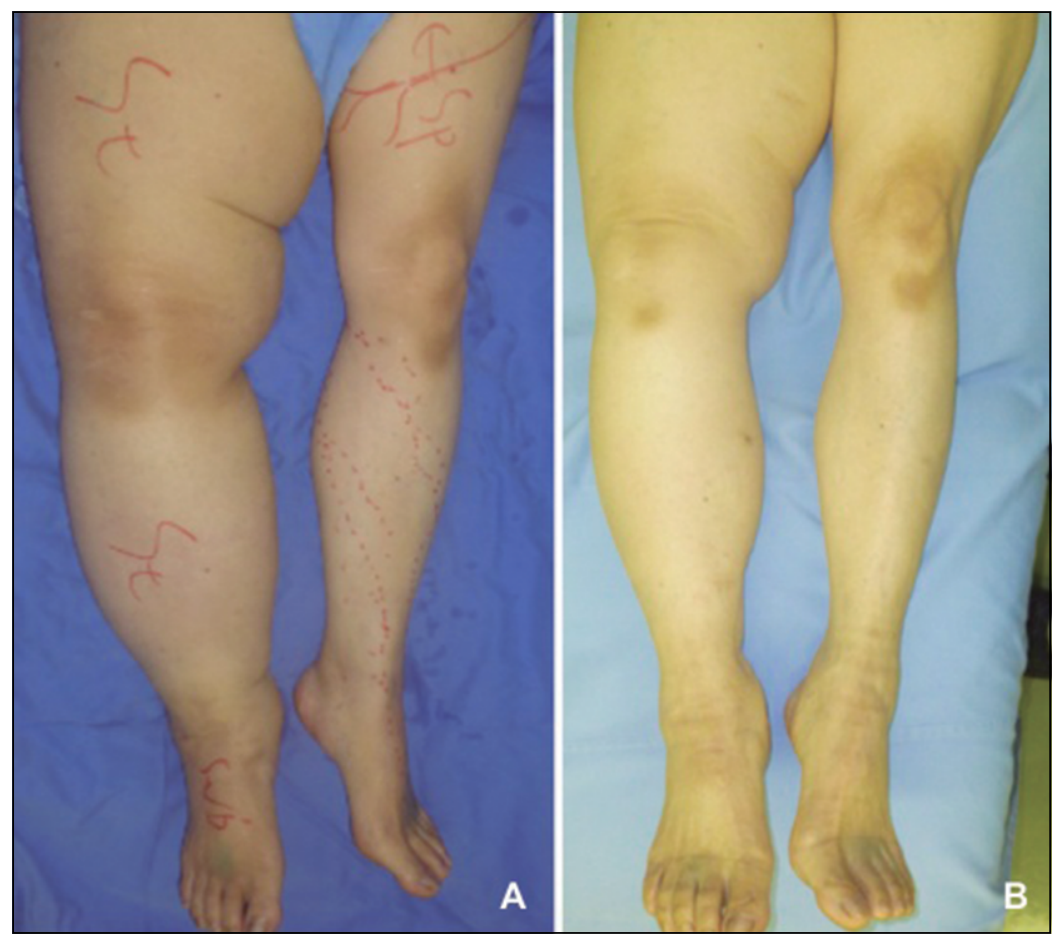

Figura 5. Paciente con linfedema de extremidad inferior derecho secundario a cáncer ginecológico. A) Imagen antes de realizar múltiples LVA; B) Imagen 15 meses posterior a la cirugía.

y constituye un desafío terapéutico. Las nuevas estrategias diagnósticas y las técnicas microquirúrgicas tienen el potencial de prevenir el desarrollo del linfedema y reconstruir fisiológicamente áreas de drenaje linfático deteriorado. El éxito depende de una buena elección de los pacientes y la realización de un tratamiento individualizado.

\section{Responsabilidades éticas}

Protección de personas y animales. Los autores declaran que para esta investigación no se han realizado experimentos en seres humanos ni en animales.

Confidencialidad de los datos. Los autores declaran que en este artículo no aparecen datos de pacientes.

Derecho a la privacidad y consentimiento informado. Los autores declaran que en este artículo no aparecen datos de pacientes.

Conflicto de intereses: no hay. 


\section{Referencias}

1. Basta MN, Gao LL, Wu LC. Operative treatment of peripheral lymphedema: a systematic meta-analysis of the efficacy and safety of lymphovenous microsurgery and tissue transplantation. Plast Reconstr Surg. 2014;133:905-13.

2. Raskin ER, Slavin SA, Borud LJ. Lymphedema. In Guyuron B, Eriksson E, Persing JA, et al, eds. Plastic Surgery: Indications and Practice. Philadelphia: Saunders Elsevier, 2009.

3. Cormier JN, Askew RL, Mungovan KS, Xing Y, Ross MI, Armer JM. Lymphedema beyond breast cancer: a systematic review and meta-analysis of cancer-related secondary lymphedema. Cancer 2010;116:5138-49.

4. Akita S, Mitsukawa N, Rikihisa N, Kubota Y, Omori N, Mitsuhashi A, et al. Early diagnosis and risk factors for lymphedema following lymph node dissection for gynecologic cancer. Plast Reconstr Surg. 2013;131:283-90.

5. Appollo K. Lower-extremity lymphedema in a patient with gynecologic cancer. Oncol Nurs Forum 2007;34:937-40.

6. Colicchia M, Sharma V, Abdollah F, Briganti A, RJ Karnes R. Therapeutic Value of Standard Versus Extended Pelvic Lymph Node Dissection During Radical Prostatectomy for HighRisk Prostate Cancer. Curr Urol Rep. 2017; 18:51.

7. Hareyama H, Hada K, Goto K, Watanabe S, Hakoyama M, Oku K, et al. Prevalence, classification, and risk factors for postoperative lower extremity lymphedema in women with gynecologic malignancies: a retrospective study. Int J Gynecol Cancer 2015;25:751-7.

8. Todo Y, Yamamoto R, Minobe S, Suzuki Y, Takeshi U, Nakatani M, et al. Risk factors for postoperative lower-extremity lymphedema in endometrial cancer survivors who had treatment including lymphadenectomy. Gynecol Oncol. 2010;119:60-4.

9. Tanaka T, Ohki N, Kojima A, Maeno Y, Miyahara Y, Sudo T, et al. Radiotherapy negates the effect of retroperitoneal nonclosure for prevention of lymphedema of the legs following pelvic lymphadenectomy for gynecological malignancies: an analysis from a questionnaire survey. Int J Gynecol Cancer 2007; 17:460-4.

10. Füller J, Guderian D, Köhler C, Schneider A, Wendt TG. Lymph edema of the lower extremities after lymphadenectomy and radiotherapy for cervical cancer. Strahlenther Onkol. 2008;184:206-11.

11. Kim JH, Choi JH, Ki EY, Lee SJ, Yoon $\mathrm{JH}$, Lee $\mathrm{KH}$, et al. Incidence and risk factors of lower-extremity lymphedema after surgery with or without adjuvant radiotherapy in patients with FIGO stage I to stage IIA cervical cancer. Int J Cancer 2012;22:686-91.

12. Mendivil AA, Rettenmaier MA, Abaid LN, Brown JV 3rd, Micha JP, Lopez $\mathrm{KL}$, et al. Lower-extremity lymphedema following management for endometrial and cervical cancer. Surg Oncol. 2016;25:200-4.

13. Taylor R, Jayasinghe UW, Koelmeyer L, Ung O, Boyages J. Reliability and validity of arm volume measurements for assessment of lymphedema. Phys Ther. 2006;86:205-14

14. Yamamoto T, Matsuda N, Todokoro T, Yoshimatsu H, Narushima M, Mihara M, et al. Lower extremity lymphedema index: a simple method for severity evaluation of lower extremity lymphedema. Ann Plast Surg. 2011;67:637-40.

15. Boccardo F, Campisi CC, Molinari L, Dessalvi S, Santi PL, Campisi C. Lymphatic complications in surgery: possibility of prevention and therapeutic options. Updates Surg. 2012;64:211-6.

16. Pereira N, Koshima I. Linfedema: Actualización en el Diagnóstico y Tratamiento Quirúrgico. Rev Chil Cir. 2018. (EN PRENSA)

17. Yamamoto $\mathrm{T}$, Narushima M, Doi K, Oshima A, Ogata F, Mihara M, et al. Characteristic indocyanine green lymphography findings in lower extremity lymphedema: the generation of a novel lymphedema severity staging system using dermal backflow patterns. Plast Reconstr Surg. 2011;127:1979-86.

18. Yamamoto T, Matsuda N, Doi K, Oshima A, Yoshimatsu H, Todokoro T, et al. The earliest finding of indocyanine green lymphography in asymptomatic limbs of lower extremity lymphedema patients secondary to cancer treatment: The modified dermal backflow stage and concept of subclinical lymphedema. Plast Reconstr Surg. 2011;128:314e-21e.

19. Akita S, Mitsukawa N, Kazama T, Kuriyama M, Kubota Y, Omori N, et al. Comparison of lymphoscintigraphy and indocyanine green lymphography for the diagnosis of extremity lymphoedema. J Plast Reconstr Aesthet Surg. 2013;66:7928.

20. Benoit L, Boichot C, Cheynel N, Arnould L, Chauffert B, Cuisenier J, et al. Preventing lymphedema and morbidity with an omentum flap after ilioinguinal lymph node dissection. Ann Surg Oncol. 200512:793-9.

21. Morotti M, Menada MV, Boccardo F, Ferrero S, Casabona F, Villa G, et al. Lymphedema microsurgical preventive healing approach for primary prevention of lower limb lymphedema after inguinofemoral lymphadenectomy for vulvar cancer. Int J Gynecol Cancer. 2013;23:769-74.

22. Boccardo F, Valenzano M, Costantini S, Casabona F, Morotti M, Sala P, et al. LYMPHA Technique to Prevent Secondary Lower Limb Lymphedema. Ann Surg Oncol. 2016;23(11):3558-63.

23. Onoda S, Todokoro T, Hara H, Azuma $\mathrm{S}$, Goto A. Minimally invasive multiple lymphaticovenular anastomosis at the ankle for the prevention of lower leg lymphedema. Microsurgery 2014;34:3726.

24. Yamamoto T, Yamamoto N, Yamashita M, Furuya M, Hayashi A, Koshima I. Efferent Lymphatic Vessel Anastomosis: Supermicrosurgical Efferent Lymphatic Vessel-to-Venous Anastomosis for the Prophylactic Treatment of Subclinical Lymphedema. Ann Plast Surg. 2016;76:424-7.

25. Iwersen LF, Sperandio FF, Toriy AM, Palú M, Medeiros da Luz C. Evidence-based practice in the management of lower limb lymphedema after gynecological cancer. Physiother Theory Pract. 2017;33:1-8.

26. Kim SJ, Park YD. Effects of complex decongestive physiotherapy on the oedema and the quality of life of lower unilateral lymphoedema following treatment for gynecological cancer. Eur J Cancer Care (Engl). 2008;17:463-8.

27. Mihara M, Hara H, Tange S, Zhou HP, Kawahara M, Shimizu Y, et al. Multisite Lymphaticovenular Bypass 
Using Supermicrosurgery Technique for Lymphedema Management in Lower Lymphedema Cases. Plast Reconstr Surg. 2016;138:262-72.

28. Seki Y, Yamamoto T, Yoshimatsu H, Hayashi A, Kurazono A, Mori M, et al. The Superior-Edge-of-the-Knee Incision Method in Lymphaticovenular Anastomosis for Lower Extremity Lymphedema. Plast Reconstr Surg. 2015; 136:665e-75e.

29. Chang DW, Suami H, Skoracki R. A prospective analysis of 100 consecutive lymphovenous bypass cases for treatment of extremity lymphedema. Plast Reconstr Surg 2013;132:1305-14.

30. Koshima I, Nanba Y, Tsutsui T, Takahashi $\mathrm{Y}$, Itoh S. Long-term follow-up after lymphaticovenular anastomosis for lymphedema in the leg. J Reconstr Microsurg. 2003;19:209-15.

31. Cormier JN, Rourke L, Crosby M, Chang $\mathrm{D}$, Armer J. The surgical treatment of lymphedema: A systematic review of the contemporary literature (2004-2010). Ann Surg Oncol. 2012;19:642-51.

32. Becker C, Assouad J, Riquet M, Hidden G. Postmastectomy lymphedema: longterm results following microsurgical lymph node transplantation. Ann Surg. 2006;243:313-5.

33. Miranda Garcés M, Mirapeix R, Pons G, Sadri A, Masià J. A Comprehensive Review of the Natural Lymphaticovenous Communications and Their Role in Lymphedema Surgery. J. Surg. Oncol. 2016;113:374-80.

34. Aschen SZ, Farias-Eisner G, Cuzzone DA, Albano NJ, Ghanta S, Weitman ES, et al. Lymph node transplantation results in spontaneous lymphatic reconnection and restoration of lymphatic flow. Plast Reconstr Surg 2014;133:301-10.

35. Dayan JH, Dayan E, Smith ML. Reverse lymphatic mapping: A new technique for maximizing safety in vascularized lymph node transfer. Plast Reconstr Surg. 2015;135:277-85.
36. Patel KM, Lin CY, Cheng MH. A prospective evaluation of lymphedemaspecific quality-of-life outcomes following vascularized lymph node transfer. Ann Surg Oncol. 2015;22:2424-30.

37. Cheng MH, Huang JJ, Nguyen DH Saint-Cyr M, Zenn MR, Tan BK, et al. A novel approach to the treatment of lower extremity lymphedema by transferring a vascularized submental lymph node flap to the ankle. Gynecol Oncol. 2012;126:93-8.

38. Batista BN, Germain M, Faria JC, Becker C. Lymph node flap transfer for patients with secondary lower limb lymphedema. Microsurgery 2017;37:29-33.

39. Sapountzis S, Ciudad P, Lim SY, Chilgar RM, Kiranantawat K, Nicoli F, et al. Modified Charles procedure and lymph node flap transfer for advanced lower extremity lymphedema. Microsurgery. 2014;34:439-47.

40. Brorson H. Liposuction in Lymphedema Treatment. J Reconstr Microsurg. 2016;32:56-65. 